\title{
Derive Urban Scale Occupant Behavior Profiles from Mobile Position Data: A Pilot Study
}

\author{
Bing Dong ${ }^{1}$, Wenbo $\mathrm{Wu}^{2}$, Qi Wang ${ }^{3}$, Sadam Abdelmutaal ${ }^{4}$, Vishnu Prakash ${ }^{4}$ \\ ${ }^{1}$ Department of Mechanical and Aerospace Engineering, \\ Syracuse University, Syracuse, New York, U.S.A. \\ ${ }^{2}$ Department of Statistics, The University of Texas at San Antonio, San Antonio, U.S.A. \\ ${ }^{3}$ Department of Civil and Environmental Engineering, Northeastern University, Boston, U.S.A. \\ ${ }^{4}$ Department of Mechanical Engineering, The University of Texas at San Antonio, \\ San Antonio, U.S.A.
}

\begin{abstract}
Occupant behavior is known as a key factor impacting building energy consumption and stochastic in nature. It is a challenge to model and simulates occupant behavior at an urban scale. This paper develops occupant behavior profile based on mobile position data for 456 buildings and compares commonly used occupant schedules from the U.S. Department of Energy Commercial Building Reference Model. In addition, a new statistical method is developed to quantify such difference. The result shows significant differences up to $50 \%$ for large office buildings and $30 \%$ for strip malls.
\end{abstract}

\section{Introduction}

People spend $90 \%$ of their time in buildings. The energy consumption of the built environment is directly related to the occupant behaviour. Therefore, the accuracy and the credibility of the occupant behaviour model directly impact the energy simulation results (Fonseca and Schlueter, 2015; Menezes et al., 2012). Similarly, the human mobility in the urban scale can improve the prediction of energy demand on the neighbourhood, district and city levels and pacify the carbon pollution caused by the build environment. Hence, it is important to understand the occupant behaviour in the urban scale. The improper selection of occupancy presence patterns could result in overestimating energy consumption in districts, which responsible for over-investment and less operational efficiency.

Urban-scale energy modeling tools have been used to 1) spatial analysis of energy consumption ( $\mathrm{Li}$ et al., 2015); 2) retrofitting energy related policy development based on the building occupancy profile (Nutkiewicz et al., 2017); 3) design and planning of new cities to optimize the energy use (Sola et al., 2018). However, most of current urban-scale energy modeling tools use pre-defined or synthetic data simulated occupancy schedules (Yan et al., 2015). None of the studies use actual occupancy schedules which is due to the lack of accurate occupancy sensors in buildings. In addition, due to the high cost of occupant counting sensors, it is almost impossible to get those information for all buildings. However, with the development of Internet of things, researchers start looking at potential data from mobile phones and Wi-Fi based sensor data (Zhou et al., 2018; Ahas et al., 2010). Recently, research on urban mobility uncover the potential to derive occupancy locations using mobile position data (Jiefan et al., 2018; Pang et al., 2018). Furthermore, geosocial network data such as Twitter, Foursquare, etc. has been used to study spatia-temporal pattern of occupants at the urban environment (Mohammadi and Taylor 2017) Riascos and Mateos (2017); Wang et al. (2018). In this paper, data collected from mobile phones is used to extract the occupancy presence patterns of more than 400 buildings at an urban scale. In addition, we categorize those buildings according to U.S. Department of Energy 16 types of building reference models (Deru et al., 2011). We compare the derived schedules with those 16 default occupancy schedules. Furthermore, a statistical method is developed to quantify the differences in these schedules. As a pilot study, this paper focuses on the analysis methodology and preliminary results with two exemplary building types as case studies.

\section{Current State-of-the-Art}

The important parameters to consider for occupancy behaviour modelling in urban scale are the location, the type and scale of the buildings. The building location is one of the most important parameters to consider for the occupancy behaviour modelling in urban scale, because the occupancy patterns differ in cities, countries and time zones (Barthelmes et al., 2018; An et al., 2017). Similar building types could follow different occupancy patterns with respect to the surrounding environment. Jiefan et al. (2018) conducted the case study for a quick service restaurant, compare the occupancy pattern of two 
buildings. One is located near highway and the other is in a community area. The result shows that the frequency of occupants more in the restaurant near by community area than that of the one near highway.

Happle (2018) discusses the importance of considering the occupant behaviour model while planning the urban building energy model. The author examines different occupancy behaviour models, such as, 1) deterministic space-based approach, 2) stochastic space-based approach and 3) stochastic person-based approach. The deterministic space-based approach is a rule-based occupant behaviour model. However, the stochastic approach is based on the occupant behaviour in built environment in different time spans. The space-based approach focuses on the whole building occupants in the building scale, while the individual-based approach studies the individual occupant behaviour. Based on the diversity of occupant behaviour, stochastic individual-based approach is superior to stochastic space-based approach. To overcome the limitation of implementing this method on both the district level and the individual building level, the author implemented a novel method "activity-based multi-agent approach". The occupant activity and the usage of appliances are monitored, which used for the urban building occupancy modelling.

General occupancy pattern detection method for urban scale modelling are commonly built upon mobile internet- based data, cellular service data, Wi-Fi based sensor data, geo-social network data such as Twitter, Foursquare, etc. The prevalence of smart-phone usage has made it possible to track the human movement and the occupancy pattern in specific area (Ahas et al., 2010). Jiefan et al. (2018) used mobile position data for modelling occupancy behaviour in urban scale. K-means clustering methodology has been applied as the data mining method to extract the typical occupancy data. The occupancy data extracted from 60 buildings are classified into 7 categories and 19 sub-categories. Different types of building show different occupancy schedules and densities. Also, the same type of building in different areas are found to follow different occupancy patterns. Pang et al. (2018) used mobile-internet based position data for modelling the occupancy behaviour in the built environment. The occupancy data were integrated with the building energy simulation (Mosteiro-Romero et al., 2017) in Energy plus, and the result shows mobile-internet based position data increases the precision accuracy of building energy model.

Mohammadi and Taylor (2017a) discusses about the spatiotemporal relationship between the human mobility and energy consumption in urban environment. They collect the twitter data and electricity consumption data for one year in the urban area of Chicago, USA. To find the spatiotemporal pattern of occupants in the urban environment, the author uses spatial autoregression model and radius of gyration is used to find the returners intra-urban mobility in the urban environment. Mohammadi and Taylor (2017b) also talks about two major network mobility patterns in the urban environment, such as returners and the explorers. The returners mobility network is dominated by the few frequently visited location. However, the explorer mobility network pattern will be much large. The energy consumption in the urban environment is not only depend on the individual building occupancy level, the author provides the scope of using spill-over effect of the neighboring building. Thus, the better understanding of mobility pattern of urban environment will help for the better prediction, management and efficient allocation of resource (Gaetani et al., 2016; Yan et al., 2015). Wang et al. (2018) talks about the urban human mobility pattern to find out the social nature of the people in 50 large American cities. The paper uses geo-social network data of Twitter to understand the human mobility pattern. Individuals tweets from each location is identified and figure out the home and work location and identifies the commute of the people in the city scale (Kim et al., 2018; Wang and Taylor, 2016). Riascos and Mateos (2017) discussed about human mobility in urban areas using locationbased social network Forursquare. The people's coincidence of meeting the people is analyzed on temporal and special basis. The social network data are used to study the occupancy behaviour pattern in the New York and Tokyo metropolitan city. The peoples travel distance and the co-incidence of meeting the people in university, bar and restaurants are discussed to find the pattern. Zhou et al. (2018) describes the different methods of collecting the crowd sensed human mobility data in city wide and building wide scaled. Firstly, the paper discusses the case study conducted for city scale and collected the human mobility pattern using the Wi-Fi based localization from students in the large city. Secondly, the author conducted case study in building wise human mobility pattern near the transportation hub. To investigate the human mobility pattern, the data collected from passive Wi-Fi tracking method. What's the key conclusion?

In summary, Urban scale occupancy behaviour model is an important parameter for the design and development of urban building energy models in neighbourhood and districts. Generally urban scale occupancy mobility model is developed using the mobile cellular devise data, mobile-internet based data, Wi-Fi based data and the geo-social network data. However, lim- 
ited data are from the geo-social network and the WiFi devices. Mobile based data set is chosen for this study.

\section{Data Sources and Handling}

\section{Data Sources}

Mobile Data The resource is smart-phone devices' GPS log dataset obtained from Cuebiq, a company that amasses large-scale location data from almost 70 million U.S. smartphones (Cuebiq, 2018). By collecting data from about 1 in 4 U.S. smartphones, Cuebiq covers almost $20 \%$ of the U.S. population. The data were collected through partner applications and relies on using modern smartphone device's internal globalpositioning system (GPS) hardware. GPS sensors log data have been previously used as a source of data in fusion frameworks to study human mobility Zheng et al. (2008) and travel mode detection (Miller, 2007) since such location data have a high spatiotemporal resolution. The raw data contain individuals recorded location information including an anonymized individual ID, latitude, longitude and the corresponding time (in seconds).

Building Information In this study, address, main use, and year built of 453 buildings in San Antonio, Texas were collected from Bexar County Appraisal District available data as shown in Figure 1. The addresses were then geocoded to their exact XY coordinates using ArcGIS. Then, these buildings information were added according to their geolocations to building footprints which was generated using ENVI. Building heights were extracted from LiDAR open source data and finally added to each building footprint. ArcGIS Polygon-to-Points tool was used to extract the vertices of each building footprint. Fi-

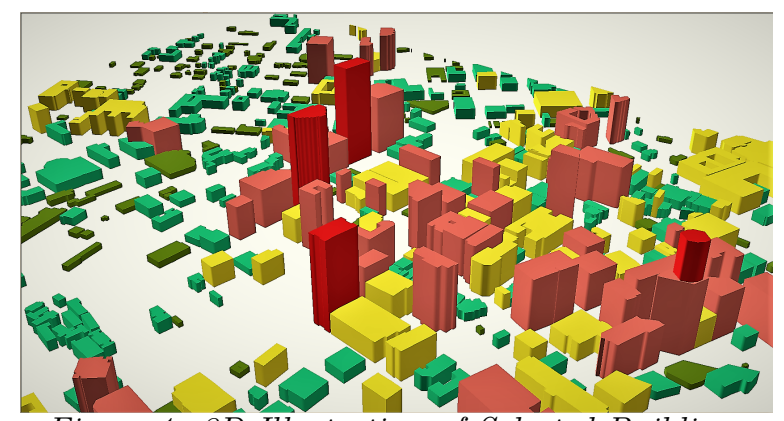

Figure 1: 3D Illustration of Selected Buildings

nally, the 453 buildings were categorized based on the DoE commercial reference buildings. Figure 2 shows the number of buildings in each DoE reference building type.

\section{Data Preparation}

The data we used for this paper focused on the users reside in the great San Antonio, TX area. The following information (variables) shown in Table 1 are recorded for each observation.

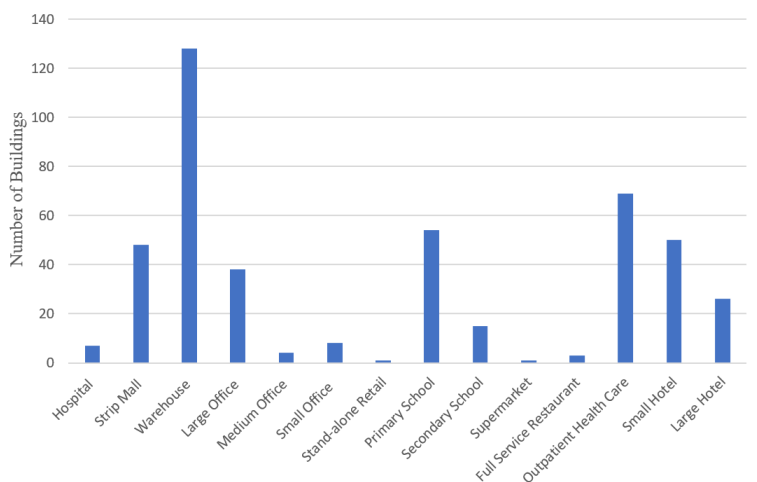

Figure 2: Number of Selected Buildings Corresponding to the DoE Reference Model Building Types

Table 1: List of variables

\begin{tabular}{|c|c|}
\hline Variables & Description \\
\hline Timestamp & UTC timestamp regarding the data point. \\
\hline Cuebiq ID & $\begin{array}{l}\text { Proprietary ID uniquely identifying each } \\
\text { device. }\end{array}$ \\
\hline IDType & Device OS representation. \\
\hline Latitude & Decimal version of the latitude. \\
\hline Longitude & Decimal version of the longitude. \\
\hline Accuracy & Data point accuracy expressed in meters. \\
\hline IP Address & $\begin{array}{l}\text { Device IP address at the time of the data } \\
\text { capture. }\end{array}$ \\
\hline Source ID & $\begin{array}{l}\text { ID uniquely representing the source of the } \\
\text { data point. }\end{array}$ \\
\hline Manufacture & The device manufacturer. \\
\hline Model & Device model. \\
\hline Carrier & The carrier code of the devices SIM card. \\
\hline Last seen & Last time of the data capture Optional. \\
\hline Country & $\begin{array}{l}\text { Country code in ISO } 3166-1 \text { alpha- } 2 \\
\text { format. }\end{array}$ \\
\hline Stat & State code. \\
\hline Zip & Zipcode related to the data point. \\
\hline
\end{tabular}

Based on these information, each user is uniquely identified by a Cuebiq ID, which is a nominal random string i.e. "ef7ff01b21c6322e8101ba8ab152ff271dec3d9dff2eae382eb5eac4c2c718d7". In this way, the user privacy is best protected while allowing maximum accessibility.

Hence, for each unique user, above information are collect at each specific time point. For better visualization and interpretation, we convert the time format from Unix Time Stamp ${ }^{1}$ into the standard "HH:MM:SS" format.

In this study, we focus on understanding the hourly occupation patterns of different buildings by tracking the occurrences of number of unique users during each hour. To achieve this goal, for each building $i$, we obtain the number of unique users $N_{i t}$ for each hour $t \in\{0,1, \ldots, 23\}$ by counting the distinct users appearing in the interior of each building using latitude and longitude of users and the building shape coordinates. We repeat this counting process for different calendar days $j$, for $j=1, \ldots, n$ where $n$ is the total number of days when data are collected. There-

\footnotetext{
${ }^{1}$ The unix time stamp is a way to track time as a running total of seconds. This count starts at the Unix Epoch on January 1st, 1970 at UTC. For example, if the time stamp of a particular observation is 1504344606 , we convert it to $09 / 02 / 2017$ at 9:30am (UTC)
} 


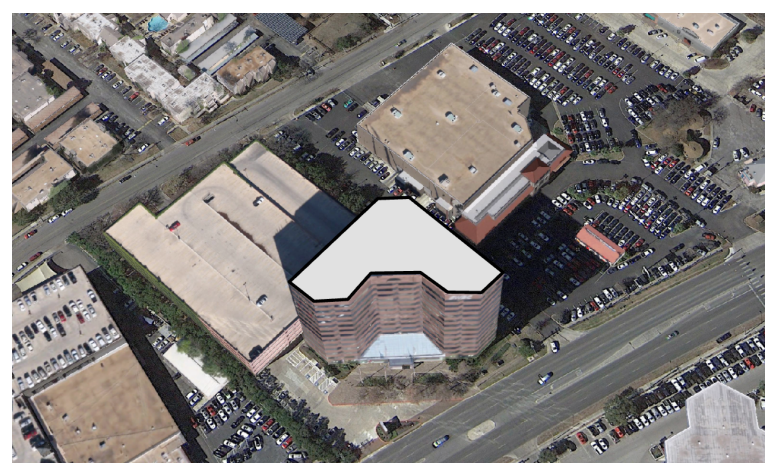

Building A (Large Office)

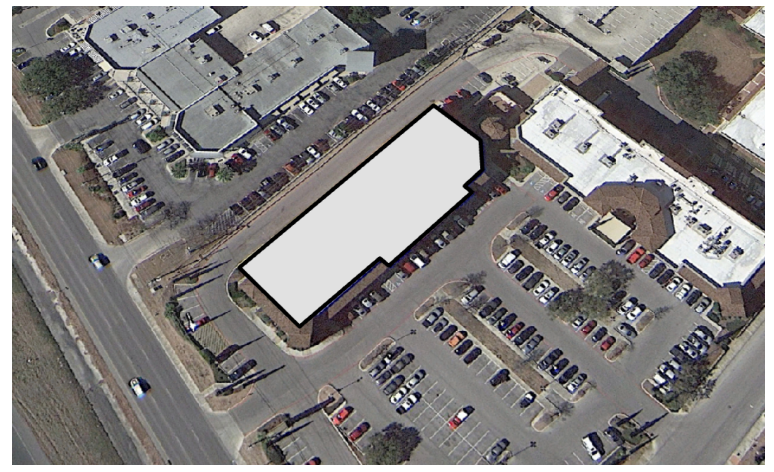

Building B (Strip Mall)

Figure 3: Building shapes

fore, we let $N_{i j t}$ denote the number of different users appear at building $i$ on day $j$ during hour $t$. Since our goal is to compare the empirical occupant presence patterns to the schedules from DoE reference models for different building types, we further split the data into three scenarios: weekdays, Saturdays, and others (Sundays and holidays) in order to make fair comparisons. Let $n_{w}, n_{s}$, and $n_{o}$ denote the total number of weekdays, Saturdays, and other days respectively so that $n_{w}+n_{s}+n_{o}=n$.

As a pilot study, in this paper we use data collected from September 2017 and an extended study using longer time span is underway.

\section{Data Visualization}

Our first goal is to visually compare the empirical hourly occupation patterns to the corresponding DoE reference patterns. In our study, we collect data for 424 buildings of 17 different types. Here, we use Building $A$ which is a large office and Building $B$ which is a strip mall as examples to illustrate our findings. We present our results for weekdays, Saturdays, and other days separately in Figure 4 and Figure 5.

The solid lines in the figures are the normalized average hourly occupations over different days for the building. To explain the process, we use weekdays as example and the calculations for other scenarios follow similarly. To normalize the hourly occupation counts, we divide the observed hourly counts $N_{i j t}$ by the empirical maximum observed counts

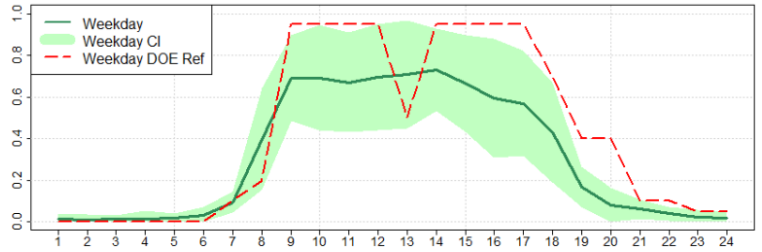

(a) Occupation Patterns for Weekdays

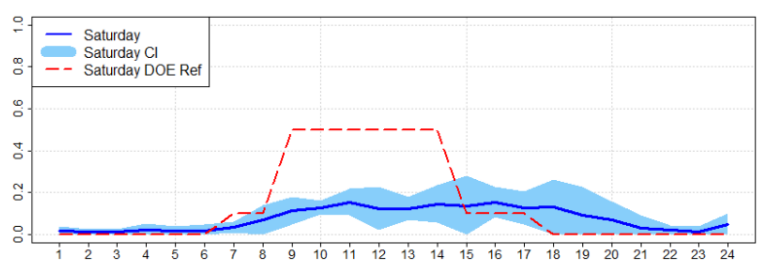

(b) Occupation Patterns for Saturdays

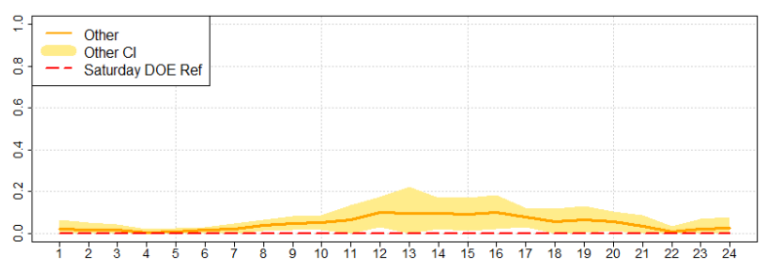

(c) Occupation Patterns for Other Days

Figure 4: Normalized Hourly Occupation Rate for Building A (Large Office).

$N_{i}^{\max }=\max _{j, h}\left\{N_{i j t}\right\}$. In this way, the normalized hourly occupation counts can be viewed as the hourly occupation rates (percentages based on building capacity). Then the average hourly occupation rates can be computed as $\bar{R}_{i t}=\frac{1}{n_{w}} \sum_{j=1}^{n_{w}} R_{i j t}$ where $R_{i j t}=N_{i j t} / N_{i}^{\max }$. The shaded band is the empirical confidence band based on the observed data for different days. Specifically, we compute the upper/lower limits of the confidence as $\bar{R}_{i t} \pm 2 \times \operatorname{std}_{i t}\left(R_{i j t}\right)$ where $\operatorname{std}_{i t}\left(R_{i j t}\right)$ is the standard deviations of $R_{\text {.jt }}$ over different weekdays. That is, for the same building we compute the standard deviations of the hourly occupation rates for the same hour over different weekdays. We use \pm 2 standard deviations to approximate the $95 \%$ confidence bands following conventional statistical study.

The red dashed lines in each plot represent the DoE hourly reference for corresponding building type at different scenarios. Loosely speaking, for any hour $t$, if the DoE reference line is outside of the confidence band generated by the empirical building occupation patterns, a "significant" discrepancy is observed. For building A, the DoE reference model follows well with the actual office building occupant presence patterns during morning arrival time and evening departure time on weekday. Interestingly, it shows differences during noon time, where the actual occupancy did not show a clear "lunch break" on weekdays. Meanwhile, building B, a strip mall, DoEs not show such break for both measured and synthetic schedules. On weekends, the DoE reference model has a huge difference with the actual schedule. However, this DoEs not happen to building $\mathrm{B}$, a shopping mall. In ad- 


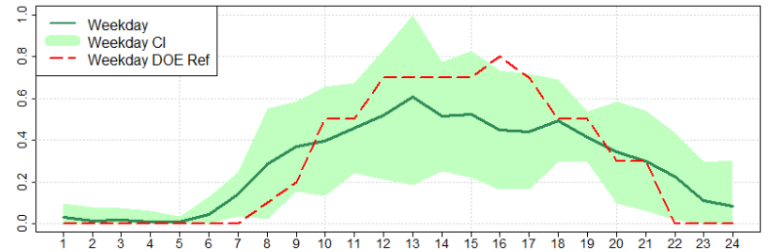

(a) Occupation Patterns for Weekdays

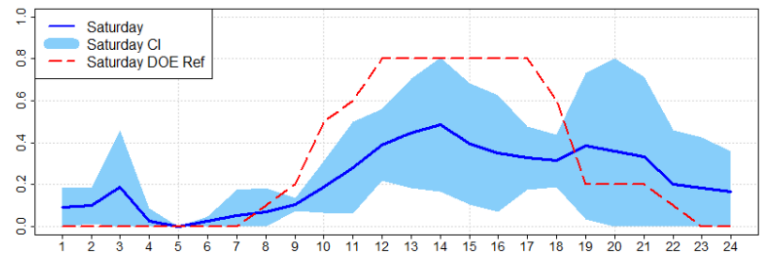

(b) Occupation Patterns for Saturdays

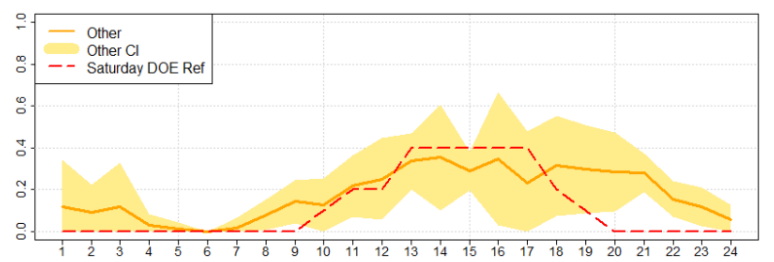

(c) Occupation Patterns for Other Days

Figure 5: Normalized Hourly Occupation Rate for Building B (Strip Mall).

dition, on both weekday and weekends, the DoE reference model schedule follows well with the actual occupancy schedule, except for a few hours on the Saturday.

\section{Statistical Analysis}

As we concluded in the previous section, we observe certain level of divergences from DoE reference patterns by the empirical hourly occupation rates. However, these divergences can take place in different ways. For example, for some buildings, the most significant different may be during the weekdays at noon while for other buildings these differences are observed during the evening hours. Hence, it is necessary to quantify the degree of divergences based on an aggregate discrepancy scores (DS). In this section, we have developed a new statistical method to compute the discrepancy score by aggregating the total differences between the DoE reference pattern and the empirical confidence limits during the 24-hour window. Specifically, we compute the discrepancy score as

$$
\mathrm{DS}_{i}=\sum_{t=0}^{23} \max \left(0, D_{i t}-U_{i t}\right)+\min \left(0, D_{i t}-L_{i t}\right),
$$

where $D_{i t}$ is the DoE reference at hour $t, U_{i t}$ and $L_{i t}$ are the upper and lower empirical confidence limits respectively at hour $t$. In other words, (1) is the aggregated areas between the DoE reference and upper confidence limit if DoE reference is above the upper confidence band and the areas between the DoE reference and lower confidence limit if DoE reference is below the confidence band. The higher DS represents a more difference the empirical hourly presence pattern to the DoE reference. Alternatively, we can also define the absolute mean discrepancy score (AMDS) by computing the total differences between the DoE references and the average hourly occupation rates $\bar{R}_{i t}$ as

$$
\operatorname{AMDS}_{i}=\sum_{t=0}^{23}\left|D_{i t}-\bar{R}_{i t}\right| .
$$

Hence, we can compute $\mathrm{DS}_{i}$ and $\mathrm{AMDS}_{i}$ for each building $i$ to quantify the difference between the empirical hourly occupation rates and DoE references. A peer-comparison on $\mathrm{DS}_{i}$ and $\mathrm{AMDS}_{i}$ of any building $i$ to the scores of other buildings of the same type can be made.

\section{Results and Discussions}

We again use SWBC Headquarters and La Arcata as examples to present our findings.

In Figure 6 we present such comparisons for Building $A$ of weekdays, Saturdays, and other days in the top, middle, and bottom panels. In the left plot, for each scenario, at each hour $t$, the box-plots display the distribution of $\max \left(0, D_{i t}-U_{i t}\right)+\min \left(0, D_{i t}-L_{i t}\right)$ for all the large offices and the dashed line represents the corresponding quantity for selected Building $A$. Specifically, each box represents the inner-quartile range, the range between the 25th percentile and 75 th percentile, of the hourly discrepancy scores for all large offices. If the dashed line lays above the box at an hour $t$, it indicates that the occupation rate of Building $A$ is much higher than the DoE reference model at hour $t$ comparing to other large offices, e.g., at $13 \mathrm{pm}$ for weekdays. Please note that any non-zero values in this plot represents a significant deviation of the observed occupancy from the DoE reference model. In other words, the DoE reference model (red dashed line) is outside of the $95 \%$ confidence band in Figure 4.

The middle plot in Figure 6 for each scenario represents the mean difference between an DoE reference model schedule at hour $t$ and the overall average occupation rates at this hour for all buildings with the same type. At each hour $t$, the box-plots display the distribution of $\bar{R}_{i t}-D_{i t}$ for all the large offices and the dashed line represents the corresponding quantity for selected Building A. A negative indicates that the observed hourly occupation rate is lower than the DoE reference model whereas a positive value is vice versa. This graph can show that how well aligned the selected office building's schedule with DoE reference model schedule. The bigger the absolute number on the $\mathrm{Y}$ axis, the larger the difference is. For example, for Building A, the observed hourly occupancy rate is much lower than the DoE reference model between $8 \mathrm{am}$ and $19 \mathrm{pm}$ except for $12 \mathrm{pm}$ where the observed 

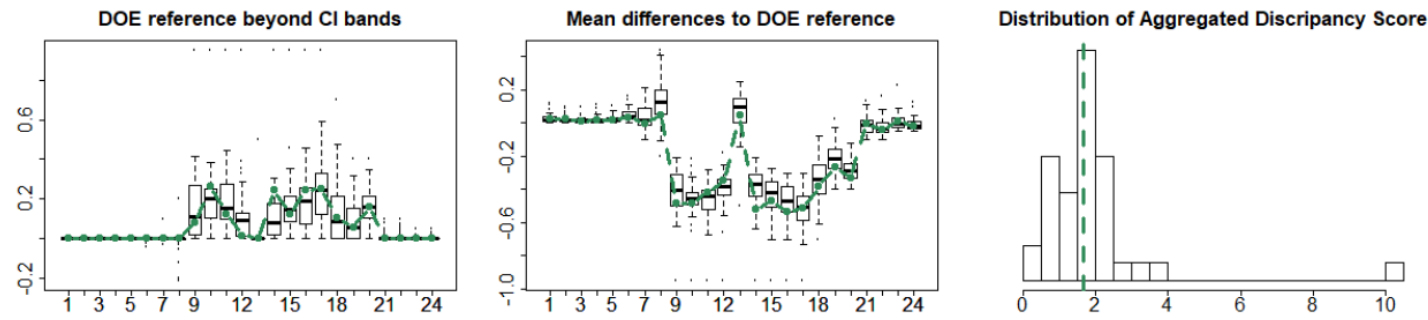

(a) Weekday
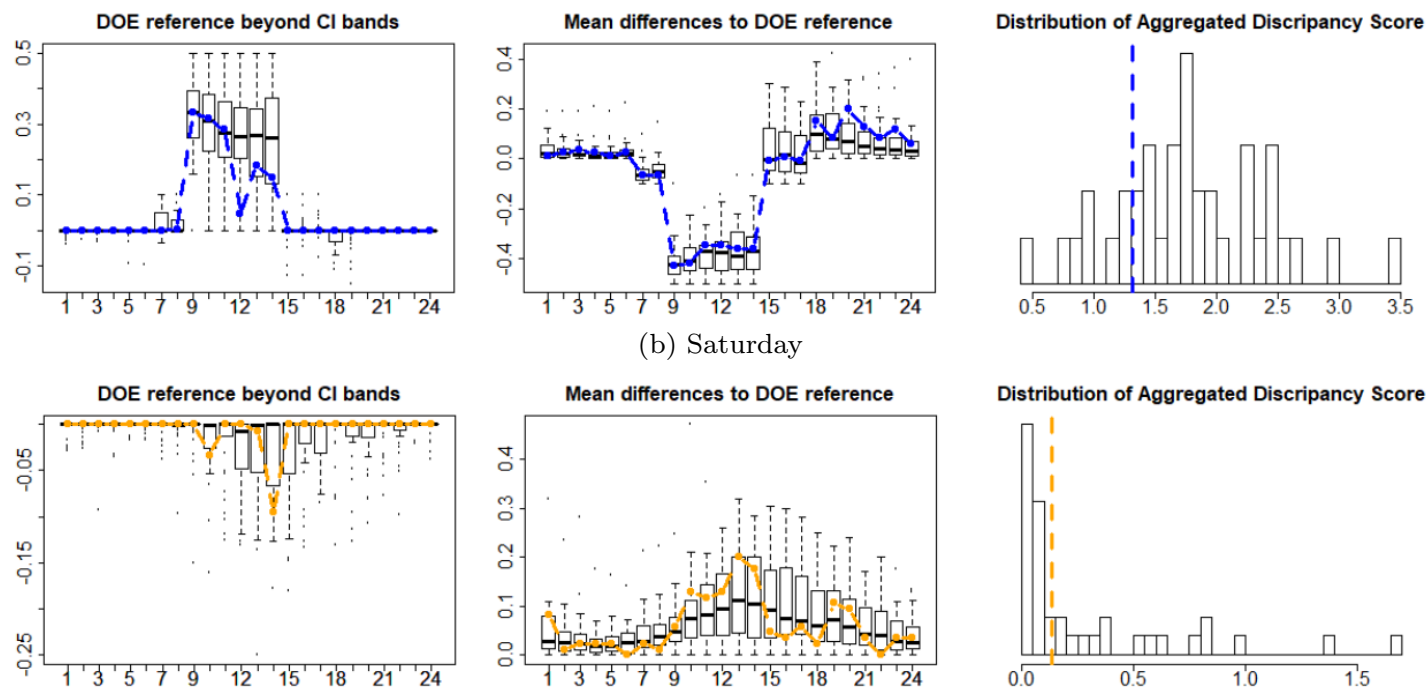

(c) Other

Figure 6: Relative Comparisons between Building A (Large Office) and other buildings with same DoE building type.
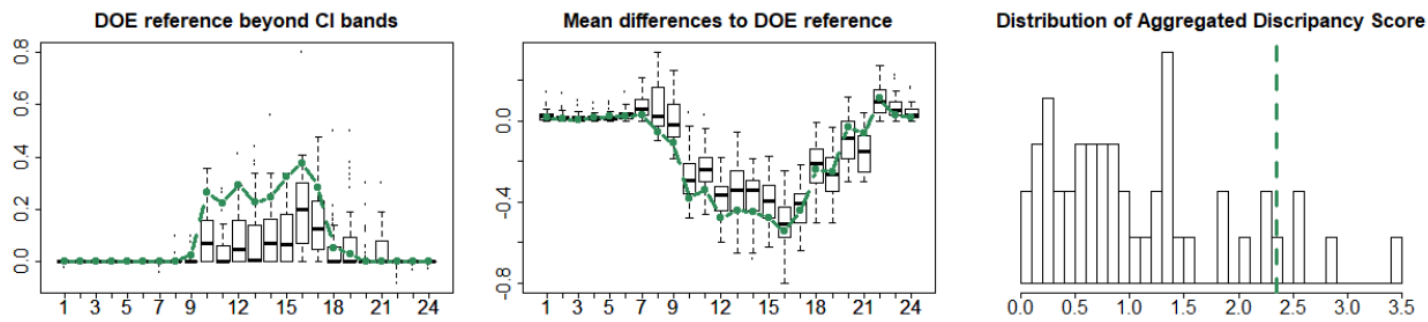

(a) Weekday
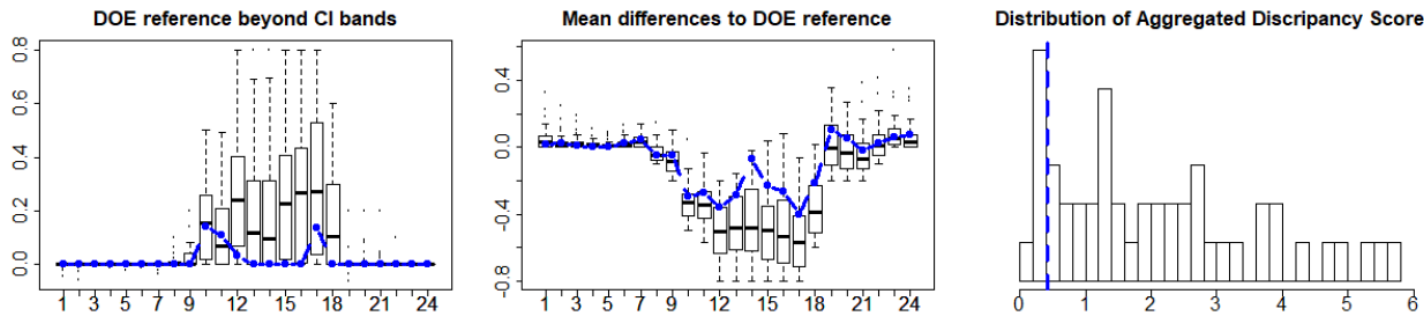

(b) Saturday
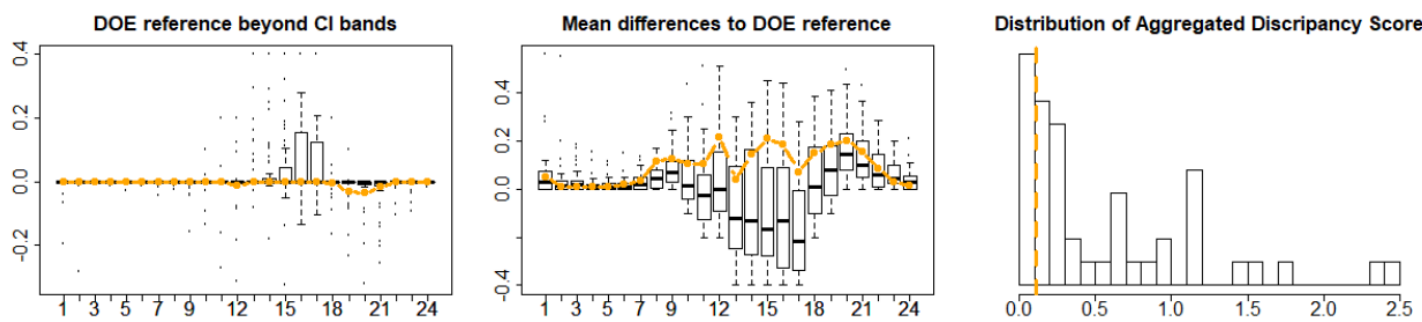

(c) Other

Figure 7: Relative Comparisons between Building B (Strip Mall) and other buildings with same DoE building type. 
Table 2: Average hourly differences (as percentage of building capacity) between measured and synthetic occupancy. The percentages in the bracket [.] denote the corresponding standard deviations of the hourly differences for the buildings of the same DoE type.

\begin{tabular}{|c|c|c|c|c|c|c|c|c|}
\hline L-O & All Day & 7am-8pm & 7 am & 8am & 9am & $6 \mathrm{pm}$ & $7 \mathrm{pm}$ & 8pm \\
\hline W & $17.70 \%[6.11 \%]$ & $29.80 \%[10.12 \%]$ & $1.63 \%[4.45 \%]$ & $8.71 \%[14.82 \%]$ & $36.72 \%$ [21.28\%] & $28.21 \%$ [21.13\%] & $18.41 \%[13.06 \%]$ & $27.73 \%[8.85 \%]$ \\
\hline $\mathrm{S}$ & $10.68 \%[1.66 \%]$ & $17.90 \%[3.45 \%]$ & $4.06 \%[4.41 \%]$ & $3.56 \%[4.12 \%]$ & $41.47 \%[8.39 \%]$ & $5.94 \%[9.54 \%]$ & $3.89 \%[7.67 \%]$ & $0.00 \%[0.00 \%]$ \\
\hline $\mathrm{O}$ & $3.73 \%[4.41 \%]$ & $5.46 \%[6.15 \%]$ & $1.72 \%[4.44 \%]$ & $2.13 \%[4.70 \%]$ & $2.26 \%[5.32 \%]$ & $4.39 \%[7.57 \%]$ & $4.89 \%[7.42 \%]$ & $4.62 \%[7.17 \%]$ \\
\hline S-M & All Day & 8am-9pm & $8 \mathrm{am}$ & 9am & 10am & $7 \mathrm{pm}$ & 8pm & 9pm \\
\hline W & $11.34 \%[5.67 \%]$ & $19.11 \%[9.77 \%]$ & $0.33 \%[1.66 \%]$ & $1.30 \%[4.08 \%]$ & $20.39 \%$ [18.09\%] & $15.85 \%[18.75 \%]$ & $5.69 \%[10.70 \%]$ & $9.83 \%[12.53 \%]$ \\
\hline $\mathrm{S}$ & $15.05 \%[7.39 \%]$ & $25.44 \%[12.84 \%]$ & $2.67 \%[4.13 \%]$ & $5.77 \%[8.10 \%]$ & $28.60 \%[16.51 \%]$ & $5.07 \%[9.75 \%]$ & $4.27 \%[7.97 \%]$ & $3.19 \%[6.83 \%]$ \\
\hline $\mathrm{O}$ & $4.87 \%[3.80 \%]$ & $7.62 \%[6.08 \%]$ & $0.68 \%[2.28 \%]$ & $3.00 \%[7.08 \%]$ & $3.35 \%[8.29 \%]$ & $5.99 \%[11.23 \%]$ & $8.03 \%[14.19 \%]$ & $7.02 \%[12.28 \%]$ \\
\hline
\end{tabular}

hourly occupancy rate is slightly higher than the DoE reference model for weekdays. For Other days, it shows higher occupancy rate than DoE reference model.

In the right plot, the histogram display the distribution of the AMDSs for all large offices and the dashed vertical line indicates where $\mathrm{AMDS}_{i}$ for Building $A$ is. Observing from the plot, for the weekdays, the overall deviation of the observed building occupancy to the DoE reference model for Building $A$ is around the average deviation to the DoE reference model for all large offices. However, the other weekdays (holidays and Sundays), the observed building occupancy rate for Building $A$ is significantly different from the DoE reference model comparing to other large offices.

Figure 7 has a very difference AMDS histogram for all weekday, Saturday and other days. This means Strip malls have very different schedules with each other. From Figure 7 middle plots of (a), (b) and (c), they all show significant deviation from the distribution of all strip malls for this selected building. This is also proved by all the right side plots.

Table 2 further illustrate the average hourly differences in schedules from measured and DoE reference model. Table 2 first shows the all day differences for large office and stripped mall are $17.7 \%$ with std of $6.1 \%$ and $11.34 \%$ with std of $5.6 \%$, respectively. In addition, we compare the differences only for day time, which are between $7 \mathrm{am}$ to $8 \mathrm{pm}$ for large office and $8 \mathrm{am}$ to $9 \mathrm{pm}$ for stripped mall. For both type of buildings, the difference significantly increased. For example, the large office schedule difference on weekday increases almost to $30 \%$ with std $10.1 \%$. This means the maximum difference of an hour is up to $50 \%$. Furthermore, we also compare the first 3 hours of arrival and last 3 hours of departure of both types of buildings. The rational is that often the first arrival and last departure time determine the operation schedule of HVAC system. For office buildings, the first one hour does not have significant difference while DoE reference models schedule shows 0.1. Starting from 7:00am, the DoE schedule is still 0.1 while the actual schedule is increasing quickly resulting a maximum difference is almost $40 \%$. During the departure time from $6 \mathrm{pm}$ to $8 \mathrm{pm}$, the deference is also significant up to $50 \%$ at $6 \mathrm{pm}$.

\section{Conclusion}

This paper presents preliminary results of a pilot study on deriving urban scale occupancy patterns at the individual building level, and compare with synthetic schedules from DoE reference models. We use two buildings as an example to illustrate our findings. The results show that for an office building there is a difference between those two schedules on both weekday and weekend, while not for a stripped mall building. In order to quantify the differences on every hour, we develop new statistical measures, discrepancy score and absolute mean discrepancy score, where former parallels the empirical statistical significance and the latter is similar to an error measure. On average, the hourly differences between measured and synthetic occupancy profile are $7.81 \%, 7.38 \%$, and $1.22 \%$ for weekdays, Saturdays, and other days respectively for large offices and $4.5 \%, 8.8 \%$, and $2.34 \%$ for weekdays, Saturdays, and other days respectively for strip malls.

The results of this study could be potentially used in 1) urban scale energy modeling: model energy consumption of each individual building with an actual measured occupancy schedule; and 2) urban mobility: predict occupancy mobility patterns to estimate the first arrival and last departure time for better building operation.

\section{Acknowledgment}

This work was supported by ARPA-E SENSOR Grant;National Science Foundation Grant HDBE1761950;Northeastern Tier 1 Project on "Neighborhood Connectivity and Social Inequality"; and Global Resilience Institute Project on "Geosocial Network Resilience". To protect the confidentiality of any given individual's movement trajectory, all individuals information from Cuebiq was encrypted, and all data are reported in unidentifiable form. The data from Cuebiq are proprietary and will not be shared.

\section{References}

Ahas, R., S. Silm, O. Järv, E. Saluveer, and M. Tiru (2010). Using mobile positioning data to model locations meaningful to users of mobile phones. Journal of Urban Technology 17(1), 3-27. 
An, J., D. Yan, T. Hong, and K. Sun (2017). A novel stochastic modeling method to simulate cooling loads in residential districts. Applied Energy 206, 134-149.

Barthelmes, V. M., R. Li, R. K. Andersen, W. Bahnfleth, S. P. Corgnati, and C. Rode (2018). Profiling occupant behaviour in danish dwellings using time use survey data - part i: Data description and activity profiling. In Proceedings of COBEE2018: 4th International Conference On Building Energy \& Environment. Melbourne (AU), 5-9 Feb 2018.

Cuebiq (2018). Cuebiq. Available: https: //www. cuebiq. com/.

National Renewable Energy Laboratory (2011). U.S. Department of Energy commercial reference building models of the national building stock.

Fonseca, J. A. and A. Schlueter (2015). Integrated model for characterization of spatiotemporal building energy consumption patterns in neighborhoods and city districts. Applied Energy 142, 247-265.

Gaetani, I., P. Hoes, and J. L. Hensen (2016). Occupant behavior in building energy simulation: Towards a fit-for-purpose modeling strategy. Applied Energy 121, 188-204.

Happle, G., F. J. A. . S. A. (2018). A review on occupant behaviour in urban building energy models. Energy and Buildings 174, 276-292.

Jiefan, G., X. Peng, P. Zhihong, C. Yongbao, J. Ying, and C. Zhe (2018). Extracting typical occupancy data of different buildings from mobile positioning data. Energy and Buildings 180, 135-145.

Kim, J., J. Park, and W. Lee (2018). Why do people move? enhancing human mobility prediction using local functions based on public records and sns data. PloS ONE 13(2).

Li, Q., S. Quan, G. Augenbroe, P. Yang, and J. Brown (2015). Building energy modeling at urban scale: Integration of reduced order energy model with geographical information. In Proceedings from IBPSA2015: 14th Conference of International Building Performance Simulation Association. Hyderabad (India),07 Dec 2015.

Menezes, A. C., A. Cripps, D. Bouchlaghem, and R. Buswell (2012). Predicted vs. actual energy performance of non-domestic buildings: Using postoccupancy evaluation data to reduce the performance gap. Applied Energy 97, 355-364.

Miller, H. (2007). Placebased versus peoplebased geographic information science. Energy and Buildings 1, 503-535.
Mohammadi, N. and J. E. Taylor (2017a). Urban energy flux: Spatiotemporal fluctuations of building energy consumption and human mobility-driven prediction. Applied Energy 195, 810-818.

Mohammadi, N. and J. E. Taylor (2017b). Urban infrastructure-mobility energy flux. Energy 140, 716-728.

Mosteiro-Romero, M., J. A. Fonseca, and A. Schlueter (2017). Seasonal effects of input parameters in urban-scale building energy simulation. Energy Procedia 122, 433-438.

Nutkiewicz, A., Z. Yang, and R. K. Jain (2017). Datadriven urban energy simulation (due-s): Integrating machine learning into an urban building energy simulation workflow. Energy Procedia 142, 21142119.

Pang, Z., P. Xu, Z. Oneill, J. Gu, S. Qiu, X. Lu, and X. Li (2018). Application of mobile positioning occupancy data for building energy simulation: An engineering case study. Building and Environment 141, 1-15.

Riascos, A. P. and J. L. Mateos (2017). Emergence of encounter networks due to human mobility. PloS ONE 12(10).

Sola, A., C. Corchero, J. Salom, and M. Sanmarti (2018). Simulation tools to build urban-scale energy models: A review. Energies 11 (12), 3269.

Wang, Q., N. E. Phillips, M. L. Small, and R. J. Sampson (2018). Urban mobility and neighborhood isolation in americas 50 largest cities. In Proceedings of the National Academy of Sciences, Volume 115, pp. 7735-7740.

Wang, Q. and J. E. Taylor (2016). Process map for urban-human mobility and civil infrastructure data collection using geosocial networking platforms. Journal of Computing in Civil Engineering 30, 04015004.

Yan, D., W. O'Brien, T. Hong, X. Feng, H. B. Gunay, F. Tahmasebi, and A. Mahdavi (2015). Occupant behavior modeling for building performance simulation: Current state and future challenges. Energy and Buildings 10\%, 264-278.

Zheng, Y., Q. Li, Y. Chen, X. Xie, and W.-Y. Ma (2008). Understanding mobility based on gps data. In Proceedings of the 10th international conference on Ubiquitous computing, pp. 312-321.

arXiv (2018). Understand Urban Human Mobility through Crowdsensed Data. 